\title{
A validation of Illumina EPIC array system with bisulfite-based amplicon sequencing
}

\author{
Alexandra Noble ${ }^{\text {Corresp., } 1}$, J F Pearson ${ }^{2}$, Joseph Boden ${ }^{3}$, John Horwood ${ }^{3}$, Neil J Gemmell ${ }^{4}$, Martin Kennedy ${ }^{2}$, Amy J \\ Osborne $^{1}$ \\ ${ }^{1}$ School of Biological Sciences, University of Canterbury, Christchurch, New Zealand \\ 2 Department of Pathology and Biomedical Sciences, University of Otago, Christchurch, New Zealand \\ 3 Department of Psychological Medicine, University of Otago, Christchurch, New Zealand \\ 4 Department of Anatomy, Univeristy of Otago, Dunedin, New Zealand \\ Corresponding Author: Alexandra Noble \\ Email address: alexandra.noble@pg.canterbury.ac.nz
}

The Illumina Infinium ${ }^{\circledR}$ MethylationEPIC BeadChip system (hereafter EPIC array) is considered to be the current gold standard detection method for assessing DNA methylation at the genome-wide level. EPIC arrays areoften used for hypothesis generation or pilot studies, the natural conclusion to which is to validate methylation candidates and expand thesein a larger cohort, in a targeted manner. As such, an accurate smaller-scale, targeted technique, that generates data at the individual CpG level that is equivalent to the EPIC array, is needed. Here, we tested an alternative DNA methylation detection technique, known as bisulfite-based amplicon sequencing (BSAS), to determine its ability to validate $\mathrm{CpG}$ sites detected in EPIC array studies. BSAS was able to detect differential DNA methylation at $\mathrm{CpG}$ sites to a degree which correlates highly with the EPIC array system at some loci. However, BSAS correlated less well with EPIC array data in some instances, and most notably, when the magnitude of change via EPIC array was greater than $5 \%$. Therefore, our data suggests that BSAS can be used to validate EPIC array data, but each locus must be compared on an individual basis, before being taken forward into large scale screening. Further, BSAS does offer advantages compared to the probe-based EPIC array; BSAS amplifies a region of the genome ( $500 \mathrm{bp})$ around aCpG of interest, allowing analyses of other CpGs in the region that may not be present on the EPIC array, aiding discovery of novel $\mathrm{CpG}$ sites and differentially methylated regions of interest. We conclude that BSAS offers a valid investigative tool for specific regions of the genome that are currently not contained on the array system. 


\section{A validation of Illumina EPIC array system with bisulfite-based amplicon}

2 sequencing

3

4 Alexandra J. Noble ${ }^{1}$, John F. Pearson ${ }^{2}$, Joseph M. Boden ${ }^{3}$, L. John Horwood ${ }^{3}$, Neil J. Gemmell ${ }^{4}$,

5 Martin A. Kennedy ${ }^{2}$, Amy J. Osborne ${ }^{1}$

6

7 Biological Sciences, University of Canterbury, Christchurch, New Zealand

8 2Department of Pathology and Biomedical Sciences, University of Otago, Christchurch, New

9 Zealand

$10{ }^{3}$ Department of Psychological Medicine, University of Otago, Christchurch, New Zealand

$11{ }^{4}$ Department of Anatomy, University of Otago, Dunedin, New Zealand

12

13 Corresponding Author:

14 Alexandra Noble

15 School of Biological Sciences, University of Canterbury, Christchurch, New Zealand

16 Email address: Alexandra.noble@pg.canterbury.ac.nz

17 


\section{Abstract}

20

21 The Illumina Infinium ${ }^{\circledR}$ MethylationEPIC BeadChip system (hereafter EPIC array) is considered

22 to be the current gold standard detection method for assessing DNA methylation at the genome23 wide level. EPIC arrays are often used for hypothesis generation or pilot studies, the natural 24 conclusion to which is to validate methylation candidates and expand these in a larger cohort, in a 25 targeted manner. As such, an accurate smaller-scale, targeted technique, that generates data at the 26 individual CpG level that is equivalent to the EPIC array, is needed. Here, we tested an alternative 27 DNA methylation detection technique, known as bisulfite-based amplicon sequencing (BSAS), to 28 determine its ability to validate $\mathrm{CpG}$ sites detected in EPIC array studies. BSAS was able to detect 29 differential DNA methylation at $\mathrm{CpG}$ sites to a degree which correlates highly with the EPIC array 30 system at some loci. However, BSAS correlated less well with EPIC array data in some instances, 31 and most notably, when the magnitude of change via EPIC array was greater than 5\%. Therefore, 32 our data suggests that BSAS can be used to validate EPIC array data, but each locus must be 33 compared on an individual basis, before being taken forward into large scale screening. Further,

34 BSAS does offer advantages compared to the probe-based EPIC array; BSAS amplifies a region 35 of the genome ( $\sim 500 \mathrm{bp})$ around a $\mathrm{CpG}$ of interest, allowing analyses of other $\mathrm{CpGs}$ in the region 36 that may not be present on the EPIC array, aiding discovery of novel CpG sites and differentially 37 methylated regions of interest. We conclude that BSAS offers a valid investigative tool for 38 specific regions of the genome that are currently not contained on the array system. 


\section{Introduction}

42

43 Epigenetic modifications, such as DNA methylation, play a vital role in regulating gene expression 44 (Hackett and Surani 2013) and have the potential to induce phenotypic changes (Dolinoy, Huang 45 et al. 2007, Sinclair, Allegrucci et al. 2007, Kucharski, Maleszka et al. 2008, Gertz, Reddy et al. 46 2012, Wang, Maurano et al. 2012). DNA methylation occurs when a methyl group is covalently 47 transferred to the C5 position of the cytosine ring of a DNA molecule by a methyltransferase 48 enzyme, with the resulting modified cytosine then termed 5-methylcytosine (5mC) (Mitchell, 49 Schneper et al. 2016). In mammals, most DNA methylation occurs at CpG dinucleotides. CpG 50 sites themselves can be defined as a singular modified cytosine residue which are found throughout 52 the genome, but are particularly dense in promoter regions (Takai and Jones 2002).

DNA methylation is heavily influenced by the surrounding environment; factors such as tobacco smoking (Breton, Byun et al. 2009, Zeilinger, Kühnel et al. 2013, Ambatipudi, Cuenin et al. 2016, Osborne, Pearson et al. 2020), alcohol (Philibert, Plume et al. 2012, Liu, Marioni et al. 2016), nutrition (Rampersaud, Kauwell et al. 2000, Delgado-Cruzata, Zhang et al. 2015), stress (Murgatroyd, Patchev et al. 2009) and aging (Horvath, Zhang et al. 2012, Marioni, Shah et al. 2015) can all impact on DNA methylation at CpG sites. Alterations to DNA methylation are associated with changes in phenotype and also, in some instances, methylation changes contribute to disease pathology (Kim, Long et al. 2010, Mastroeni, Grover et al. 2010, Rakyan, Beyan et al. 2011, De Jager, Srivastava et al. 2014).

As a result of these relatively recent observations, the assessment of differential DNA methylation in humans, and in particular, epigenome-wide association studies (EWAS), is a burgeoning field. High-throughput array technologies are a popular choice for EWAS, due to their robustness and accuracy (Pidsley, Zotenko et al. 2016). The Illumina Infinium ${ }^{\circledR}$ MethylationEPIC array (hereafter 'EPIC array') quantifies methylation at 850,000 different CpG sites (Zhou, Laird et al. 2017), and although this is still a small proportion of the total number of $\mathrm{CpG}$ sites in the genome $(\sim 28$ million (Lövkvist, Dodd et al. 2016)) it represents a broad distribution of sites that give a specific and robust measurement of methylation at those sites. 
72 Further, the goal of many whole-genome studies of DNA methylation is often a pilot or scoping 73 study to capture a range of targets that may be associating with, e.g., a particular environmental 74 exposure. As such, once the genome has been investigated in a number of samples, a whole75 genome approach is not always necessary if the user simply requires follow up and/or validation 76 of identified loci in a larger cohort. To undertake further analyses and to validate methylation 77 array-based experiments, several different methods exist that that rely on bisulfite treatment of 78 DNA: bisulfite-based amplicon sequencing (BSAS), bisulfite pyrosequencing and methylation79 specific PCR (MS-PCR) are methods which can specifically target a predetermined area of interest 80 in the genome at a low cost and higher sample throughput, compared to arrays. An informative study conducted by the BLUEPRINT consortium evaluated 27 predefined genomic regions, across 32 reference samples amongst 18 laboratories using six assays (Bock, Halbritter et al. 2016). Good agreement was observed across methods, with amplicon bisulfite sequencing, and bisulfite pyrosequencing showing the best concordance (Bock, Halbritter et al. 2016). A similar study also assessed bisulfite pyrosequencing, observing congruence to EPIC array analysis (Roessler, Ammerpohl et al. 2012). However, pyrosequencing is known to have quantitative flaws due to the output of sequences generated through fluorescence methods (França, Carrilho et al. 2002). MS-PCR is a method often used in clinical settings (Herman, Graff et al. 1996), however it has a high false positive rate (Claus, Wilop et al. 2012). By contrast, BSAS detects cytosine methylation to base-pair scale resolution without reliance on light detection methods for sequencing (Masser,

Stanford et al. 2015). BSAS is a multiplex procedure that can quantitatively assess each CpG site within numerous target regions at the same time (Masser, Berg et al. 2013).

Thus, given the limitations of pyrosequencing and MS-PCR, here we examine whether BSAS can also accurately validate EPIC array data, and be used as a replication, and/or expansion tool for targeted DNA methylation analyses, similar to what has been shown using pyrosequencing. Further, we wish to assess the multiple other $\mathrm{CpG}$ sites residing within the targeted amplicon region, to investigate differential methylated regions, which would not be able to be explored via EPIC array.

To answer the question, we used EPIC array data generated from individuals from the Christchurch 
103 response to regular cannabis use (Osborne, Pearson et al. 2020). The CHDS is a longitudinal study 104 of a birth cohort of 1265 children born in 1977 in Christchurch, New Zealand, who have been 105 studied on 24 occasions from birth to the age of $40(n=904$ at age 40$)$. Of this, a total 96 individuals 106 were selected, and arrays were performed in two separate batches in consecutive years $(n=48$ per 107 year).

108

For validation analysis we selected individuals with EPIC array data $(n=14)$, as well as new individuals $(\mathrm{n}=82)$, to serve as a validation and expansion cohort for the differential DNA methylation identified via EPIC array (Osborne, Pearson et al. 2020). Specifically, we asked whether BSAS, after determination of the most appropriate normalisation method, produced the same average methylation values as EPIC arrays, when comparing case data to control data. While both EPIC array and BSAS are readily used as standalone experiments, they would provide robust evidence if carried out together. Thus, given the rising popularity of studies investigating DNA methylation, establishing a better understanding of how differential DNA methylation differs between regions within the genome, such as evaluating concordance between methods and then further assessing resultant $\mathrm{CpG}$ sites within a designated region, is valuable to the scientific community.

\section{Materials \& Methods}

124

\section{Cohort selection and DNA extraction - EPIC arrays}

EPIC array data used in this study has previously been published (Osborne, Pearson et al. 2020). Briefly, in this study we use DNA from human participants who are partitioned into three groups: i) regular cannabis users, who had never used tobacco ("cannabis-only"); those who consumed both cannabis and tobacco ("cannabis plus tobacco"), and; iii) controls, who consumed neither cannabis nor tobacco. Controls were matched as closely as possible for sex, ethnicity and parental socioeconomic status (data and methods described in (Osborne, Pearson et al. 2020)).

\section{Bioinformatics analysis - processing and normalisation of raw EPIC array data}


134 For this study, analysis was carried out using R statistical software (Version 3.5.2) (Team 2013).

135 Quality control was first performed on the raw data; sex chromosomes and 150 failed probes 136 (detection $\mathrm{P}$ value greater than 0.01 in at least 50\% of samples) were excluded from analysis. 137 Furthermore, potentially problematic CpGs with adjacent single nucleotide polymorphisms 138 (SNPs), or that did not map to a unique location in the genome (Pidsley, Zotenko et al. 2016) were 139 also excluded. The raw data were then normalised using Noob (Fortin, Triche et al. 2017) in the 140 minfi package (Aryee, Jaffe et al. 2014). Normalisation was then checked by observing density 141 plots as well as multidimensional scaling plots of the 5000 most variable $\mathrm{CpG}$ sites.

142

143 Cohort selection and DNA extraction - BSAS experiments

144 BSAS analysis was carried out on two groups: cannabis plus tobacco users $(n=44)$ and controls $145(\mathrm{n}=38)$, who had never used cannabis. In contrast to the EPIC array analysis, no cannabis-only 146 participants were used in BSAS; this is a consequence of the small number of individuals who use 147 cannabis but who do not also use tobacco. Cannabis users were all selected on the basis that they 148 either met DSM-IV diagnostic criteria (Association 2000) for cannabis dependence or had reported using cannabis consumption on a daily basis for a minimum of three years prior to age 28 . Participants were matched as closely as possible for the following variables, sex, ethnicity, and parental socioeconomic status (Supplementary Table 1). All participants were collected across a four month period so they are all of a similar age. Collection and analysis of DNA in the Christchurch Health and Development Study was approved by Southern Health and Disability Ethics Committee (CTB/04/11/234/AM10). DNA extraction protocols are previously described in (Noble, Pearson et al. 2020). Specifically, DNA was extracted from whole blood samples using a Kingfisher Flex System (Thermo Scientific, Waltham, MA USA) and quantified via nanodrop 157 (Thermo Scientific, Waltham, MA USA). DNA was bisulfite treated using the EZ DNA 158 Methylation-Gold kit (Zymo Research, USA) as per the manufacturer's instructions.

159

\section{CpG site selection, primer design and amplification - BSAS}

A total of $15 \mathrm{CpG}$ sites, representing 15 individual probes from the Illumina EPIC array were chosen based on their differential methylation status in cannabis plus tobacco users compared to controls (Table 1). A range of probes at differing levels of significance (not significant, nominally 
165 by the EPIC arrays. Primers to amplify bisulfite-treated DNA were designed using the online tool 166 BiSearch (Arányi, Váradi et al. 2006) to amplify a 250 base pair region which spanned the CpG 167 site (Supplementary Table 2). At the 5' end of each primer sequence, an Illumina overhang (33 168 base pair sequence) was included to ensure the ability to pool the amplicons and barcode them for 169 high-throughput sequencing. All product lengths were all between 226 and 340 base pairs. To 170 ensure primer specificity, Delta G's were designed to be no lower than $-9 \mathrm{kcal} / \mathrm{mol}$ for efficiently, 171 using the tool OligoAnalyzer (IDT®). A total of 30 primer pairs were initially designed for this 172 experiment, and 15 of these are discussed here, as these were the primer pairs which performed 173 efficiently at first usage. PCRs were undertaken as per (Noble, Pearson et al. 2020).

174

175 Following the PCR, DNA was cleaned up with Agencourt ${ }^{\circledR}$ AMPure ${ }^{\circledR}$ XP beads (Beckman 176 Coulter) and washed with $80 \%$ ethanol and allowed to air-dry. DNA was then eluted with $52.5 \mu 1$ 177 of $10 \mathrm{mM}$ Tris $\mathrm{pH} 8.5$ before being placed back into the magnetic stand. Once the supernatant had 178 cleared, $50 \mu \mathrm{l}$ of supernatant was taken up and aliquotted into a fresh 96-well plate. DNA samples 179 were quantified using the Quant-iT ${ }^{\mathrm{TM}}$ PicoGreen $^{\mathrm{TM}}$ dsDNA Assay kit (Thermo Fisher) using the 180 FLUROstar ${ }^{\circledR}$ Omega (BMG Labtech). Sequence libraries were prepared using the Illumina MiSeq $^{\mathrm{TM}} 500$ cycle Kit V2, and sequenced on an Illumina $\mathrm{MiSeq}^{\mathrm{TM}}$ system at Massey Genome Services (Palmerston North, New Zealand).

183

\section{Bioinformatic and statistical analysis - BSAS data}

185 Illumina MiSeq ${ }^{\mathrm{TM}}$ sequences were trimmed using SolexQA++ software and aligned to FASTA 186 bisulfite converted reference sequences using the package Bowtie2 (version 2.3.4.3). Each 187 individual read was then aligned to all reference sequences (GRCh37/hg19) using the methylation188 specific package Bismark (Krueger and Andrews 2011). Bismark produced aligned mapped reads 189 with counts for methylated and unmethylated cytosines at each CpG site, thus BSAS returns 190 additional $\mathrm{CpG}$ sites to the intended validation target, as each sequencing read contains multiple 191 CpG sites. Cytosine proportion is calculated based upon the number of cytosines divided by the 192 number of cytosines with the additions of the number of thymines present $\left(C /\left(C_{1}\right)+T\right)$. This gave

193 the average methylation $\beta$ values for each individual at each given $\mathrm{CpG}$ site. These $\beta$ values could 194 be anywhere between 0 - 1, with a $\beta$ equal to 1 indicating $100 \%$ methylation at that $\mathrm{CpG}$ site across 195 all sequencing reads. These data were imported into R Studio (RStudio version 3.3.0) and the 
196 edgeR package (Chen, Pal et al. 2017) was used to determine differential DNA methylation 197 between cannabis users and controls; coverage level was set to greater or equal to " 8 " across 198 unmethylated and methylated counts. This was also set at 50 and 100 reads and no differences 199 were seen between the results at any of these thresholds, so " 8 " was used for the continuation of BSAS calling under the recommendations of (Chen, Pal et al. 2017) whereby the conservative rule 201 of thumb is total count (both methylated and unmethylated is at least " 8 " in every sample. Within 202 the data set $96.5 \%$ of the reads were above a methylation coverage of 50 (Supplementary data file). 203 A negative binomial generalised model was used to fit the counts (methylated and unmethylated 204 reads) in regards to the two variable groups. Summary tables compiled of the $\mathrm{CpG}$ sites of interest 205 with nominal $\mathrm{P}$ value significance and post multiple testing using false discovery rate (FDR) of 206 less than 0.05 were considered to be statistically significant. A scatter plot including a linear 207 regression line with adjusted $\mathrm{R}^{2}$ values was generated in $\mathrm{R}$ Studio to quantify the correlation between $\beta$ values produced with EPIC array and BSAS. Adjusted $\mathrm{R}^{2}$ values were calculated for: i) BSAS cases versus EPIC cases, and; ii) BSAS controls versus EPIC controls. A Bland Altman analysis (Martin Bland and Altman 1986) was used to compare the agreement of the two techniques. Means were log transformed and lower and upper levels of agreement with $95 \%$ 212 confidence intervals were calculated. Welch two sample t-tests were carried out on each of the loci 213 (cases and controls separately) to assess differences between the two methods. All graphs were 214 constructed using the R package ggplot2 (Wickham 2016).

215

216

217

218

\section{Results}

219

\section{Validation and replication of EPIC array data using BSAS:}

221

222

The differences between average methylation ( $\beta$ values) of cannabis plus tobacco users (cases) and controls were calculated for each method (EPIC array and BSAS, Table 1).

224

225

When comparing case vs control data from EPIC and BSAS individually, no significant difference 226 in average methylation between case and control was observed for either detection method, with 227 the exception of $\operatorname{cg} 05575921$ in $A H R R$ and $\operatorname{cg} 09078959 ; A H R R$ was significant in both BSAS and 
228 EPIC $\left(\mathrm{P}=0.006, \mathrm{P}=5.33 \times 10^{-12}\right)$, and $\operatorname{cg} 05575921$ was found to only be significant under BSAS $229(\mathrm{P}=0.001, \mathrm{P}=0.665)$.

230

231

232 Correlations between BSAS and EPIC were plotted individually for cases and controls. BSAS 233 versus EPIC cases resulted in an adjusted $\mathrm{R}^{2}$ of 0.8878 and BSAS versus EPIC controls gave an 234 adjusted $\mathrm{R}^{2}$ of 0.8683 (Supplementary Figure 1).

Bland Altman correlation

237

A Bland Altman analysis was carried out on the loci investigated by BSAS and compared to data 239 for the same loci produced using the Illumina EPIC array. Figure 1A shows cannabis users (cases) measured using BSAS and the EPIC array on the X axis, while the $\mathrm{Y}$ axis represents the $\log$ differences between the measurements. The observed differences between loci in cannabis cases (EPIC and BSAS) fall within the lines of agreement. Figure 1B shows the control group differences plotted for the same loci for BSAS and the EPIC array methods. Similar to above, all data points fall within the lower and upper lines of agreement.

Mean methylation values for each individual were plotted for each of the 15 loci, and these were then compared between BSAS and EPIC, for cases (Figure 2) and controls (Figure 3). Loci displaying a significant shift in average methylation between the methods of detection are indicated with an * when using a Welsh two sample comparison. The following loci were found to display differences between BSAS and EPIC array: cases; AHRR, $\operatorname{cg} 09078959, \operatorname{cg} 11293828$, CHD7, DP10, EHMT2 and ITPR1, and controls; AHRR, cg09078959, cg11293828, CHD7, DP10, EHMT2, ITPR1, NIPAL4 and PPMIL.

\section{Assessing amplicon regions}

Multiple $\mathrm{CpG}$ sites residing within an amplicon can be sequenced using BSAS, providing 259 information about a larger region of interest, rather than just a single CpG site. Supplementary 
260 Figure 2 displays the multiple CpG sites found across each of the 15 amplicons in this study. A 261 total of 9 of the 15 amplicons contained more than one $\mathrm{CpG}$ site. All $\mathrm{CpG}$ sites within the 262 amplicons remained non-differentially methylated between cases and controls, except one site in 263 AHRR. The amplicon from SLC17A7 sequenced here contained a total of $15 \mathrm{CpG}$ sites with in the 264250 base pairs.

265

266

267 Discussion

268

269

High throughput array technologies have facilitated the next step in assessing associations between DNA methylation changes in response to a known environmental exposure at a genome-wide 271 level. The EPIC array (as well as the predecessor $27 \mathrm{k}$ and $450 \mathrm{k}$ arrays) is one such platform that

272 273 274 275

276 277 278 279 280 281 282 283 284 285 286 287 288 289 290 allows for the characterisation of these DNA methylation changes. Through these approaches, various studies have furthered our understanding of how DNA methylation can play a role in response to different environmental exposures.

We selected the orthogonal method BSAS to determine its applicability as a validation, replication and/or expansion tool for EPIC array. BSAS is often used as a standalone method for assessing differential DNA methylation at specific $\mathrm{CpG}$ sites, usually because it is more cost-effective than EPIC arrays, and allows analysis of many samples at once, in multiplex. It returns data for all CpGs within a targeted region of interest ( $\sim 250$ base pairs) with results providing base pair-level specificity (Masser, Stanford et al. 2015). Overall, when considering average methylation between cases and controls as determined via BSAS or EPIC individually, we did not detect significant differences in average methylation for each detection method; the biological results are discussed elsewhere (Osborne, Pearson et al. 2020), however, it was expected that the smaller sample set used here would not have the statistical power to detect effects found in the larger cohort. The intent of this study was to compare average methylation as determined via BSAS, to that determined by EPIC array. We show here that the estimation of differential DNA methylation observed using BSAS correlated with differential methylation determined via EPIC array. However, although the data correlates between the methods (adjusted $\mathrm{R}^{2}$ cases, 0.8878 and adjusted $\mathrm{R}^{2}$ controls, 0.8683 ), we urge caution when interpreting this correlation as proof that 
291 BSAS will be a suitable independent validation of EPIC array data in every experiment. This is 292 because while the data presented here correlated between BSAS and EPIC array as a whole dataset, 293 some sites showed larger differences between average methylation estimated using BSAS vs. EPIC 294 array. Most notably, where the differential methylation on EPIC array was greater than 5\% 295 between cases and controls, BSAS was unable to detect this differential DNA methylation to the 296 same magnitude as EPIC array. Further, a total of 9/15 loci had observed P value significance

297 298 299 300 301 302 303 304 305 306 307 308 309 310 when carrying out a Welch two sample t-test on control data, and 7/15 on case data, implying there were differences between the methylation values for the methods. For instance, $A H R R$ exhibited a $4 \%$ difference in methylation between cases and controls when assessed using BSAS (the highest value detected in using BSAS in this study), compared to $23 \%$ using EPIC array. Thus, while a strong correlation between EPIC array data and BSAS data was found across the $15 \mathrm{CpG}$ sites investigated, which itself implies an association between the average methylation at each $\mathrm{CpG}$ for the two techniques, each locus must be validated on a case by case basis before being taken forward into high-throughput or large scale screening, to ensure it produces results that are equivalent to EPIC. In addition, further work on $\mathrm{CpG}$ sites with higher magnitude changes is needed to determine whether BSAS is limited by the magnitude of differential methylation it is able to detect. However, it is worth noting that most studies of differential methylation report modest $(<5 \%)$ significant differential methylation observations, suggesting that BSAS may prove useful, given inclusion of rigorous controls of known differential methylation to ensure accuracy of results.

Due to the sequence-based nature of BSAS data (compared to the probe-based nature of EPIC arrays) BSAS, as a standalone method, offers some advantages that are not applicable to EPIC arrays. For instance, BSAS has the potential to determine novel differentially methylated CpGs which may be near (in the same targeted region) but not the initial pre-determined CpG site of interest. This is possible because all CpGs within an, e.g., 500bp region are returned using BSAS data, only one of which may be on an EPIC array. Further, via this targeted sequencing process, BSAS may reveal novel differentially methylated regions (DMRs). DMRs are described as areas which exhibit multiple successive methylated $\mathrm{CpG}$ sites which may have biological impact within the genome. For example, they have been implicated in the development and progression of disease (Hotta, Kitamoto et al. 2018). Therefore targeting more than a single CpG site may provide further insight into genes and regions of interest. Consequently, while here we 
322 have used BSAS technology to replicate/validate differential methylation identified via EPIC

323 array, given that BSAS outputs can correlate with EPIC data, equally, BSAS could be used as a

324 "discovery-based tool"; if significantly differentially methylated CpGs are identified via BSAS,

325 this would serve to justify further investigation using a robust and more expensive high

326 throughout method.

327

328

329 The EPIC array still remains the most reproducible way to measure DNA methylation (Bibikova, 330 Le et al. 2009). This is because the probe-based nature of the method frequently produces 331 comparable results across research groups and arrays. For example, detection of differential 332 methylation using the EPIC array found a difference of $23 \%$ in cannabis plus tobacco users, 333 compared to controls, at AHRR (cg05575921, Table 1), a result that is supported by other studies 334 in tobacco smokers using EPIC array (Zeilinger, Kühnel et al. 2013, Guida, Sandanger et al. 2015, 335 Ambatipudi, Cuenin et al. 2016, Su, Wang et al. 2016, Li, Wong et al. 2018). AHRR has an 336 important role in controlling a range of different physiological functions; it contributes to regulation of cell growth, regulation of apoptosis and contributes to vascular and immune responses (Trombino, Near et al. 2000, Allan and Sherr 2005, Lahvis, Pyzalski et al. 2005, Marlowe, Fan et al. 2008).

340

341

BSAS and EPIC array rely upon different chemistries and methods to detect DNA methylation.

342 This may account for the majority of the variation found between the two methods. BSAS relies 343 upon PCR amplification of DNA that is treated with sodium bisulfite. When DNA is treated, 344 unmethylated cytosine residues are converted into uracil via hydrolytic deamination. 345 Amplification of uracil nucleotides during this process are replaced by thymine during replication 346 and the 5-methylcytosines are left unreactive throughout the deamination process and then are 347 amplified as cytosines. It then becomes possible to 'read' values of methylation for each cytosine 348 in an amplicon via DNA sequencing (Booth, Ost et al. 2013). The ability to treat DNA with sodium 349 bisulfite has led to the expansion of research undertaken within this field (Frommer, McDonald et 350 al. 1992). However, it is important that we ensure the validity of the results are not limited by the 351 manner in which the data was produced. Ensuring that we limit these discrepancies between technologies will allow for better validation of data. There is potential for errors to occur at this 
353 step, because incomplete bisulfite conversion cannot be distinguished from 5-methylcytosine, this 354 can possibly introduce false positive methylation calls at this point (Richards, Patel et al. 2018) 355 (Krueger, Kreck et al. 2012). Although both techniques rely upon bisulfite treatment, it is this 356 source of error followed by the PCR amplification that might explain the differences in results we 357 have observed. Refining these sources of error may provide much more comparable results 358 between the two methods.

359

360

361

362

363

364

365

366

\section{Conclusions}

In conclusion, we chose to validate EPIC array data by using the alternative method, BSAS, to detect differential methylation at $\mathrm{CpG}$ sites. While BSAS validated EPIC array data at some loci, and correlated across all loci as a whole, some individual loci did not validate. This implies that each locus must be investigated individually before determining its utility in a large-scale analysis. Further, it is possible that BSAS may be unable to reproduce the magnitude of changes that are shown in the EPIC array system, which may be a consequence of lack of specificity and addition error rate through PCR amplification. It does however, have the ability to assess differentially methylated regions, rather than individual $\mathrm{CpG}$ sites. As some regions of the genome are more susceptible to methylation change than others, BSAS could detect swathes of correlated differential methylation at neighbouring $\mathrm{CpG}$ sites in certain areas of the genome. From the results shown here, BSAS has the potential to be able to detect methylation marks which maybe hallmarks

374 for disease later on in life. Finally, although BSAS does not generate the same significance level 375 as the EPIC array in some instances, we demonstrate that BSAS can be used as an investigative tool for specific regions of the genome.

377

378

\section{Acknowledgements}

379

University of Otago Research Grant to M.A.K., The Carney Centre for Pharmacogenomics. CHDS was funded by the Health Research Council of New Zealand (Programme Grant 16/600) and the Canterbury Medical Research Foundation. 
385

386

387

388

389

390

391

392

393

394

395

396

397

398

399

400

401

402

403

404

405

406

407

408

409

410

411

412

413

414

415

416

417

418

419

420

421

422

423

424

425

426

427

428

429

\section{References}

Allan, L. L. and D. H. Sherr (2005). "Constitutive activation and environmental chemical induction of the aryl hydrocarbon receptor/transcription factor in activated human B lymphocytes." Mol Pharmacol 67(5): 1740-1750.

Ambatipudi, S., C. Cuenin, H. Hernandez-Vargas, A. Ghantous, F. L. Calvez-Kelm, R. Kaaks, M. Barrdahl, H. Boeing, K. Aleksandrova, A. Trichopoulou, P. Lagiou, A. Naska, D. Palli, V. Krogh, S. Polidoro, R. Tumino, S. Panico, B. Bueno-de-Mesquita, P. H. Peeters, J. R. Quirós, C. Navarro, E. Ardanaz, M. Dorronsoro, T. Key, P. Vineis, N. Murphy, E. Riboli, I. Romieu and Z. Herceg (2016). "Tobacco smoking-associated genome-wide DNA methylation changes in the EPIC study." Epigenomics 8(5): 599-618.

Ambatipudi, S., C. Cuenin, H. Hernandez-Vargas, A. Ghantous, F. Le Calvez-Kelm, R. Kaaks, M. Barrdahl, H. Boeing, K. Aleksandrova and A. Trichopoulou (2016). "Tobacco smoking-associated genome-wide DNA methylation changes in the EPIC study." Epigenomics 8(5): 599-618.

Arányi, T., A. Váradi, I. Simon and G. E. Tusnády (2006). "The BiSearch web server." BMC Bioinformatics 7(1): 431.

Aryee, M. J., A. E. Jaffe, H. Corrada-Bravo, C. Ladd-Acosta, A. P. Feinberg, K. D. Hansen and R. A. Irizarry (2014). "Minfi: a flexible and comprehensive Bioconductor package for the analysis of Infinium DNA methylation microarrays." Bioinformatics (Oxford, England) 30(10): 1363-1369.

Association, A. P. (2000). Diagnostic criteria from dsM-iV-tr, American Psychiatric Pub.

Bibikova, M., J. Le, B. Barnes, S. Saedinia-Melnyk, L. Zhou, R. Shen and K. L. Gunderson (2009). "Genomewide DNA methylation profiling using Infinium ${ }^{\circledR}$ assay." Epigenomics 1(1): 177-200.

Bock, C., F. Halbritter, F. J. Carmona, S. Tierling, P. Datlinger, Y. Assenov, M. Berdasco, A. K. Bergmann, K. Booher, F. Busato, M. Campan, C. Dahl, C. M. Dahmcke, D. Diep, A. F. Fernández, C. Gerhauser, A. Haake, K. Heilmann, T. Holcomb, D. Hussmann, M. Ito, R. Kläver, M. Kreutz, M. Kulis, V. Lopez, S. S. Nair, D. S. Paul, N. Plongthongkum, W. Qu, A. C. Queirós, F. Reinicke, G. Sauter, T. Schlomm, A. Statham, C. Stirzaker, R. Strogantsev, R. G. Urdinguio, K. Walter, D. Weichenhan, D. J. Weisenberger, S. Beck, S. J. Clark, M. Esteller, A. C. Ferguson-Smith, M. F. Fraga, P. Guldberg, L. L. Hansen, P. W. Laird, J. I. MartínSubero, A. O. H. Nygren, R. Peist, C. Plass, D. S. Shames, R. Siebert, X. Sun, J. Tost, J. Walter, K. Zhang and B. c. The (2016). "Quantitative comparison of DNA methylation assays for biomarker development and clinical applications." Nature Biotechnology 34(7): 726-737.

Booth, M. J., T. W. B. Ost, D. Beraldi, N. M. Bell, M. R. Branco, W. Reik and S. Balasubramanian (2013). "Oxidative bisulfite sequencing of 5-methylcytosine and 5-hydroxymethylcytosine." Nature Protocols 8 : 1841.

Breton, C. V., H.-M. Byun, M. Wenten, F. Pan, A. Yang and F. D. Gilliland (2009). "Prenatal tobacco smoke exposure affects global and gene-specific DNA methylation." American journal of respiratory and critical care medicine 180(5): 462-467.

Chen, Y., B. Pal, J. E. Visvader and G. K. Smyth (2017). "Differential methylation analysis of reduced representation bisulfite sequencing experiments using edgeR." F1000Research 6: 2055-2055.

Claus, R., S. Wilop, T. Hielscher, M. Sonnet, E. Dahl, O. Galm, E. Jost and C. Plass (2012). "A systematic comparison of quantitative high-resolution DNA methylation analysis and methylation-specific PCR." Epigenetics 7(7): 772-780.

De Jager, P. L., G. Srivastava, K. Lunnon, J. Burgess, L. C. Schalkwyk, L. Yu, M. L. Eaton, B. T. Keenan, J. Ernst, C. McCabe, A. Tang, T. Raj, J. Replogle, W. Brodeur, S. Gabriel, H. S. Chai, C. Younkin, S. G. Younkin, F. Zou, M. Szyf, C. B. Epstein, J. A. Schneider, B. E. Bernstein, A. Meissner, N. Ertekin-Taner, L. B. Chibnik, 
430 M. Kellis, J. Mill and D. A. Bennett (2014). "Alzheimer's disease: early alterations in brain DNA

431 methylation at ANK1, BIN1, RHBDF2 and other loci." Nature Neuroscience 17(9): 1156-1163.

432 Delgado-Cruzata, L., W. Zhang, J. A. McDonald, W. Y. Tsai, C. Valdovinos, L. Falci, Q. Wang, K. D. Crew, R. 433 M. Santella, D. L. Hershman and H. Greenlee (2015). "Dietary modifications, weight loss, and changes in 434 metabolic markers affect global DNA methylation in Hispanic, African American, and Afro-Caribbean 435 breast cancer survivors." J Nutr 145(4): 783-790.

436 Dolinoy, D. C., D. Huang and R. L. Jirtle (2007). "Maternal nutrient supplementation counteracts 437 bisphenol A-induced DNA hypomethylation in early development." Proceedings of the National 438 Academy of Sciences 104(32): 13056-13061.

439 Fortin, J.-P., T. J. Triche, Jr. and K. D. Hansen (2017). "Preprocessing, normalization and integration of the 440 Illumina HumanMethylationEPIC array with minfi." Bioinformatics (Oxford, England) 33(4): 558-560.

441 França, L. T., E. Carrilho and T. B. Kist (2002). "A review of DNA sequencing techniques." Quarterly 442 reviews of biophysics 35(2): 169-200.

443 Frommer, M., L. E. McDonald, D. S. Millar, C. M. Collis, F. Watt, G. W. Grigg, P. L. Molloy and C. L. Paul 444 (1992). "A genomic sequencing protocol that yields a positive display of 5-methylcytosine residues in 445 individual DNA strands." Proc Natl Acad Sci U S A 89(5): 1827-1831.

446 Gertz, J., T. E. Reddy, K. E. Varley, M. J. Garabedian and R. M. Myers (2012). "Genistein and bisphenol A 447 exposure cause estrogen receptor 1 to bind thousands of sites in a cell type-specific manner." Genome 448 research 22(11): 2153-2162.

449 Guida, F., T. M. Sandanger, R. Castagné, G. Campanella, S. Polidoro, D. Palli, V. Krogh, R. Tumino, C. 450 Sacerdote, S. Panico, G. Severi, S. A. Kyrtopoulos, P. Georgiadis, R. C. H. Vermeulen, E. Lund, P. Vineis 451 and M. Chadeau-Hyam (2015). "Dynamics of smoking-induced genome-wide methylation changes with 452 time since smoking cessation." Human Molecular Genetics 24(8): 2349-2359.

453 Hackett, J. A. and M. A. Surani (2013). "DNA methylation dynamics during the mammalian life cycle." 454 Philosophical Transactions of the Royal Society B: Biological Sciences 368(1609): 20110328.

455 Herman, J. G., J. R. Graff, S. Myohanen, B. D. Nelkin and S. B. Baylin (1996). "Methylation-specific PCR: a 456 novel PCR assay for methylation status of CpG islands." Proc Natl Acad Sci U S A 93(18): 9821-9826. Horvath, S., Y. Zhang, P. Langfelder, R. S. Kahn, M. P. Boks, K. van Eijk, L. H. van den Berg and R. A. Ophoff (2012). "Aging effects on DNA methylation modules in human brain and blood tissue." Genome Biology 13(10): R97.

Hotta, K., A. Kitamoto, T. Kitamoto, Y. Ogawa, Y. Honda, T. Kessoku, M. Yoneda, K. Imajo, W. Tomeno, S. Saito and A. Nakajima (2018). "Identification of differentially methylated region (DMR) networks associated with progression of nonalcoholic fatty liver disease." Scientific Reports 8(1): 13567. Kim, M., T. I. Long, K. Arakawa, R. Wang, M. C. Yu and P. W. Laird (2010). "DNA Methylation as a Biomarker for Cardiovascular Disease Risk." PLOS ONE 5(3): e9692.

465 Krueger, F. and S. R. Andrews (2011). "Bismark: a flexible aligner and methylation caller for Bisulfite-Seq 466 applications." Bioinformatics 27(11): 1571-1572.

467 Krueger, F., B. Kreck, A. Franke and S. R. Andrews (2012). "DNA methylome analysis using short bisulfite 468 sequencing data." Nature Methods 9: 145.

469 Kucharski, R., J. Maleszka, S. Foret and R. Maleszka (2008). "Nutritional control of reproductive status in 470 471 honeybees via DNA methylation." Science 319(5871): 1827-1830.

Lahvis, G. P., R. W. Pyzalski, E. Glover, H. C. Pitot, M. K. McElwee and C. A. Bradfield (2005). "The aryl hydrocarbon receptor is required for developmental closure of the ductus venosus in the neonatal mouse." Mol Pharmacol 67(3): 714-720.

474 Li, S., E. M. Wong, M. Bui, T. L. Nguyen, J.-H. E. Joo, J. Stone, G. S. Dite, G. G. Giles, R. Saffery, M. C. 475 Southey and J. L. Hopper (2018). "Causal effect of smoking on DNA methylation in peripheral blood: a 476 twin and family study." Clinical epigenetics 10: 18-18. 
477 Liu, C., R. E. Marioni, Å. K. Hedman, L. Pfeiffer, P. C. Tsai, L. M. Reynolds, A. C. Just, Q. Duan, C. G. Boer, T. 478 Tanaka, C. E. Elks, S. Aslibekyan, J. A. Brody, B. Kühnel, C. Herder, L. M. Almli, D. Zhi, Y. Wang, T. Huan, C. 479 Yao, M. M. Mendelson, R. Joehanes, L. Liang, S. A. Love, W. Guan, S. Shah, A. F. McRae, A. Kretschmer, 480 H. Prokisch, K. Strauch, A. Peters, P. M. Visscher, N. R. Wray, X. Guo, K. L. Wiggins, A. K. Smith, E. B. 481 Binder, K. J. Ressler, M. R. Irvin, D. M. Absher, D. Hernandez, L. Ferrucci, S. Bandinelli, K. Lohman, J. Ding, 482 L. Trevisi, S. Gustafsson, J. H. Sandling, L. Stolk, A. G. Uitterlinden, I. Yet, J. E. Castillo-Fernandez, T. D. 483 Spector, J. D. Schwartz, P. Vokonas, L. Lind, Y. Li, M. Fornage, D. K. Arnett, N. J. Wareham, N.

484 Sotoodehnia, K. K. Ong, J. B. J. van Meurs, K. N. Conneely, A. A. Baccarelli, I. J. Deary, J. T. Bell, K. E. 485 North, Y. Liu, M. Waldenberger, S. J. London, E. Ingelsson and D. Levy (2016). "A DNA methylation 486 biomarker of alcohol consumption." Molecular Psychiatry 23: 422.

487 Lövkvist, C., I. B. Dodd, K. Sneppen and J. O. Haerter (2016). "DNA methylation in human epigenomes 488 depends on local topology of CpG sites." Nucleic Acids Research 44(11): 5123-5132.

489 Marioni, R. E., S. Shah, A. F. McRae, B. H. Chen, E. Colicino, S. E. Harris, J. Gibson, A. K. Henders, P. 490 Redmond, S. R. Cox, A. Pattie, J. Corley, L. Murphy, N. G. Martin, G. W. Montgomery, A. P. Feinberg, M. 491 D. Fallin, M. L. Multhaup, A. E. Jaffe, R. Joehanes, J. Schwartz, A. C. Just, K. L. Lunetta, J. M. Murabito, J. 492 M. Starr, S. Horvath, A. A. Baccarelli, D. Levy, P. M. Visscher, N. R. Wray and I. J. Deary (2015). "DNA 493 methylation age of blood predicts all-cause mortality in later life." Genome Biology 16(1): 25.

494 Marlowe, J. L., Y. Fan, X. Chang, L. Peng, E. S. Knudsen, Y. Xia and A. Puga (2008). "The aryl hydrocarbon receptor binds to E2F1 and inhibits E2F1-induced apoptosis." Mol Biol Cell 19(8): 3263-3271. Martin Bland, J. and D. Altman (1986). "STATISTICAL METHODS FOR ASSESSING AGREEMENT BETWEEN TWO METHODS OF CLINICAL MEASUREMENT." The Lancet 327(8476): 307-310.

Masser, D. R., A. S. Berg and W. M. Freeman (2013). "Focused, high accuracy 5-methylcytosine quantitation with base resolution by benchtop next-generation sequencing." Epigenetics \& Chromatin 6(1): 33.

Masser, D. R., D. R. Stanford and W. M. Freeman (2015). "Targeted DNA methylation analysis by nextgeneration sequencing." Journal of visualized experiments : JoVE(96): 52488.

Mastroeni, D., A. Grover, E. Delvaux, C. Whiteside, P. D. Coleman and J. Rogers (2010). "Epigenetic changes in Alzheimer's disease: Decrements in DNA methylation." Neurobiology of Aging 31(12): 20252037.

Mitchell, C., L. M. Schneper and D. A. Notterman (2016). "DNA methylation, early life environment, and health outcomes." Pediatr Res 79(1-2): 212-219.

Murgatroyd, C., A. V. Patchev, Y. Wu, V. Micale, Y. Bockmühl, D. Fischer, F. Holsboer, C. T. Wotjak, O. F. $X$. Almeida and D. Spengler (2009). "Dynamic DNA methylation programs persistent adverse effects of early-life stress." Nature Neuroscience 12: 1559.

Noble, A. J., J. F. Pearson, A. D. Noble, J. M. Boden, L. J. Horwood, M. A. Kennedy and A. J. Osborne (2020). "Epigenetic signatures associated with the observed link between maternal tobacco use during pregnancy, and offspring conduct problems in childhood and adolescence." bioRxiv: 2020.2007.2002.183285.

Osborne, A. J., J. F. Pearson, A. J. Noble, N. J. Gemmell, L. J. Horwood, J. M. Boden, M. C. Benton, D. P. Macartney-Coxson and M. A. Kennedy (2020). "Genome-wide DNA methylation analysis of heavy cannabis exposure in a New Zealand longitudinal cohort." Translational Psychiatry 10(1): 114. Philibert, R. A., J. M. Plume, F. X. Gibbons, G. H. Brody and S. R. H. Beach (2012). "The impact of recent alcohol use on genome wide DNA methylation signatures." Frontiers in genetics 3: 54-54. Pidsley, R., E. Zotenko, T. J. Peters, M. G. Lawrence, G. P. Risbridger, P. Molloy, S. Van Djik, B. Muhlhausler, C. Stirzaker and S. J. Clark (2016). "Critical evaluation of the Illumina MethylationEPIC BeadChip microarray for whole-genome DNA methylation profiling." Genome biology 17(1): 208-208. 
523 Pidsley, R., E. Zotenko, T. J. Peters, M. G. Lawrence, G. P. Risbridger, P. Molloy, S. Van Djik, B.

524 Muhlhausler, C. Stirzaker and S. J. Clark (2016). "Critical evaluation of the Illumina MethylationEPIC

525 BeadChip microarray for whole-genome DNA methylation profiling." Genome Biology 17(1): 208.

526 Rakyan, V. K., H. Beyan, T. A. Down, M. I. Hawa, S. Maslau, D. Aden, A. Daunay, F. Busato, C. A. Mein, B.

527

528

529

530

531

532

533

534

535

536

537

538

539

540

541

542

543

544

545

546

547

548

549

550

551

552

553

554

555

556

557

558

559

560

561

562

563

564 Manfras, K.-R. M. Dias, C. G. Bell, J. Tost, B. O. Boehm, S. Beck and R. D. Leslie (2011). "Identification of Type 1 Diabetes-Associated DNA Methylation Variable Positions That Precede Disease Diagnosis." PLOS Genetics 7(9): e1002300.

Rampersaud, G. C., G. P. Kauwell, A. D. Hutson, J. J. Cerda and L. B. Bailey (2000). "Genomic DNA methylation decreases in response to moderate folate depletion in elderly women." The American Journal of Clinical Nutrition 72(4): 998-1003.

Richards, R., J. Patel, K. Stevenson and S. Harbison (2018). "Evaluation of massively parallel sequencing for forensic DNA methylation profiling." Electrophoresis 39(21): 2798-2805.

Roessler, J., O. Ammerpohl, J. Gutwein, B. Hasemeier, S. L. Anwar, H. Kreipe and U. Lehmann (2012). "Quantitative cross-validation and content analysis of the 450k DNA methylation array from Illumina, Inc." BMC Research Notes 5(1): 210.

Sinclair, K. D., C. Allegrucci, R. Singh, D. S. Gardner, S. Sebastian, J. Bispham, A. Thurston, J. F. Huntley, W. D. Rees and C. A. Maloney (2007). "DNA methylation, insulin resistance, and blood pressure in offspring determined by maternal periconceptional B vitamin and methionine status." Proceedings of the National Academy of Sciences 104(49): 19351-19356.

Su, D., X. Wang, M. R. Campbell, D. K. Porter, G. S. Pittman, B. D. Bennett, M. Wan, N. A. Englert, C. L. Crowl, R. N. Gimple, K. N. Adamski, Z. Huang, S. K. Murphy and D. A. Bell (2016). "Distinct Epigenetic Effects of Tobacco Smoking in Whole Blood and among Leukocyte Subtypes." PLOS ONE 11(12): e0166486.

Takai, D. and P. A. Jones (2002). "Comprehensive analysis of CpG islands in human chromosomes 21 and 22." Proceedings of the National Academy of Sciences 99(6): 3740-3745.

Team, R. C. (2013). R: A language and environment for statistical computing. R Foundation for Statistical Computing. Vienna, Austria.

Trombino, A. F., R. I. Near, R. A. Matulka, S. Yang, L. J. Hafer, P. A. Toselli, D. W. Kim, A. E. Rogers, G. E. Sonenshein and D. H. Sherr (2000). "Expression of the aryl hydrocarbon receptor/transcription factor (AhR) and AhR-regulated CYP1 gene transcripts in a rat model of mammary tumorigenesis." Breast Cancer Res Treat 63(2): 117-131.

Wang, H., M. T. Maurano, H. Qu, K. E. Varley, J. Gertz, F. Pauli, K. Lee, T. Canfield, M. Weaver and R. Sandstrom (2012). "Widespread plasticity in CTCF occupancy linked to DNA methylation." Genome research 22(9): 1680-1688.

Wickham, H. (2016). ggplot2: Elegant Graphics for Data Analysis, Springer-Verlag New York. Zeilinger, S., B. Kühnel, N. Klopp, H. Baurecht, A. Kleinschmidt, C. Gieger, S. Weidinger, E. Lattka, J. Adamski, A. Peters, K. Strauch, M. Waldenberger and T. Illig (2013). "Tobacco Smoking Leads to Extensive Genome-Wide Changes in DNA Methylation." PLOS ONE 8(5): e63812. Zhou, W., P. W. Laird and H. Shen (2017). "Comprehensive characterization, annotation and innovative use of Infinium DNA methylation BeadChip probes." Nucleic acids research 45(4): e22-e22. 


\section{Table $\mathbf{1}$ (on next page)}

CPG site differences from EPIC array and the BSAS methods at the 15 loci of differing levels of significance (not significant, nominally significant, and significant after P-value adjustment) 
1 Table 1: CpG site differences from EPIC array and the BSAS methods at the 15 loci of 2 differing levels of significance (not significant, nominally significant, and significant after 3 P-value adjustment)

\begin{tabular}{|c|c|c|c|c|c|c|c|c|c|c|}
\hline & & & & Illur & ina EPIC & ray & & BSAS & & \\
\hline & Cg/Gene & $\begin{array}{l}\text { Position in } \\
\text { genome }\end{array}$ & Illumina ID & $\begin{array}{r}\beta \\
\text { difference }\end{array}$ & $\mathrm{P}$ value & $\begin{array}{r}\text { FDR } \\
\text { Adjusted P } \\
\text { value }\end{array}$ & $\begin{array}{r}\beta \\
\text { difference }\end{array}$ & $\begin{array}{r}P \\
\text { value }\end{array}$ & $\begin{array}{r}\text { FDR } \\
\text { Adjusted P } \\
\text { value }\end{array}$ & $\beta$ difference \\
\hline 1 & $A H R R$ & Chr5, GB & cg05575921 & -0.233 & 5.33E-12 & $3.7 \mathrm{E}-06$ & -0.041 & $0.006^{*}$ & 0.245 & -0.192 \\
\hline 2 & cg11977356* & Chr19 & cg11977356 & -0.040 & 0.474 & 0.999 & -0.004 & 0.406 & 0.959 & -0.036 \\
\hline 3 & ITPR1 & Chr3, GB & cg08987995 & -0.001 & 0.572 & 0.999 & 0.005 & 0.820 & 0.822 & -0.006 \\
\hline 4 & MAGI & Chr7, GB & cg21121803 & -0.008 & 0.572 & 0.999 & -0.007 & 0.809 & 0.959 & -0.0004 \\
\hline 5 & EHMT2 & Chr6, GB & cg07829740 & 0.005 & 0.037 & 0.999 & -0.015 & 0.071 & 0.579 & 0.020 \\
\hline 6 & PPM1L & Chr3, GB & cg26406186 & -0.006 & 0.818 & 0.999 & 0.011 & 0.904 & 0.963 & -0.017 \\
\hline 7 & cg00571101* & Chr12 & cg00571101 & 0.004 & 0.368 & 0.999 & -0.004 & 0.813 & 0.952 & 0.008 \\
\hline 8 & cg09078959* & Chr5 & cg09078959 & -0.001 & 0.893 & 0.999 & -0.005 & $0.001^{*}$ & 0.245 & 0.004 \\
\hline 9 & cg01614625* & Chr7 & cg01614625 & -0.009 & 0.370 & 0.999 & -0.006 & 0.569 & 0.952 & -0.004 \\
\hline 10 & $D P 10$ & Chr2, GB & cg05868547 & 0.006 & 0.077 & 0.999 & -0.003 & 0.713 & 0.952 & 0.009 \\
\hline 11 & cg11293828* & Chr12 & cg11293828 & -0.014 & 0.665 & 0.999 & 0.032 & 0.735 & 0.952 & -0.045 \\
\hline 12 & CHD7 & $\begin{array}{l}\text { Chr5, } \\
\text { 5'UTR }\end{array}$ & cg19926587 & -0.007 & 0.960 & 0.999 & -0.006 & 0.429 & 0.959 & -0.001 \\
\hline 13 & NIPAL4 & $\begin{array}{l}\text { Chr5, } \\
\text { TSS1500 }\end{array}$ & cg17695979 & -0.007 & 0.714 & 0.999 & -0.003 & 0.106 & 0.713 & -0.004 \\
\hline 14 & PRDM5 & Chr4, GB & cg01118724 & -0.004 & 0.734 & 0.999 & 0.005 & 0.116 & 0.713 & -0.009 \\
\hline 15 & SLC17A7 & Chr19, GB & cg02624701 & -0.043 & 0.312 & 0.999 & 0.018 & 0.646 & 0.952 & -0.061 \\
\hline
\end{tabular}

5

Table 1 *When a cg number is listed, then there is no known gene associated with that CPG site. GB-Gene Body

6 


\section{Figure 1}

Figure 1 Bland Altman of BSAS vs EPIC for cases and controls

Bland Altman plots showing the log mean differences between DNA methylation as measured by EPIC array vs. the same CpG sites measured using BSAS. A) Data from cannabis users, gathered using BSAS and the EPIC array (Cases) B) the control subjects used in BSAS and EPIC array. Each of the 15 points represents the CpG sites investigated. Dotted lines represent the limits of agreement, red the mean, blue in the $95 \%$ confidence intervals.

A

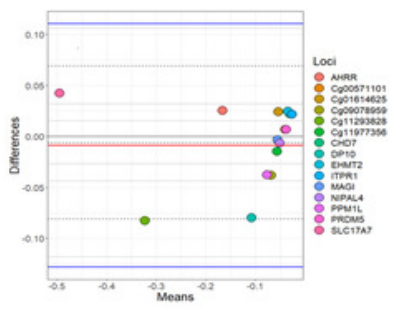

B

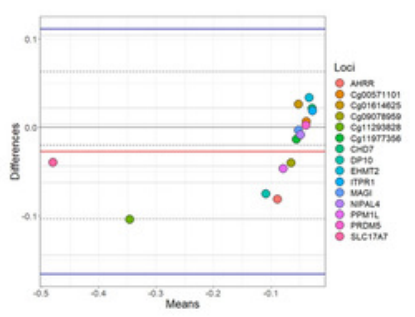


Figure 2

Figure 2 Individual methylation across the 15 loci for cases

Figure 2- Average methylation for case individuals only across the 15 loci assessed using EPIC and BSAS .* represent those loci with significant differences in average methylation between EPIC and BSAS.

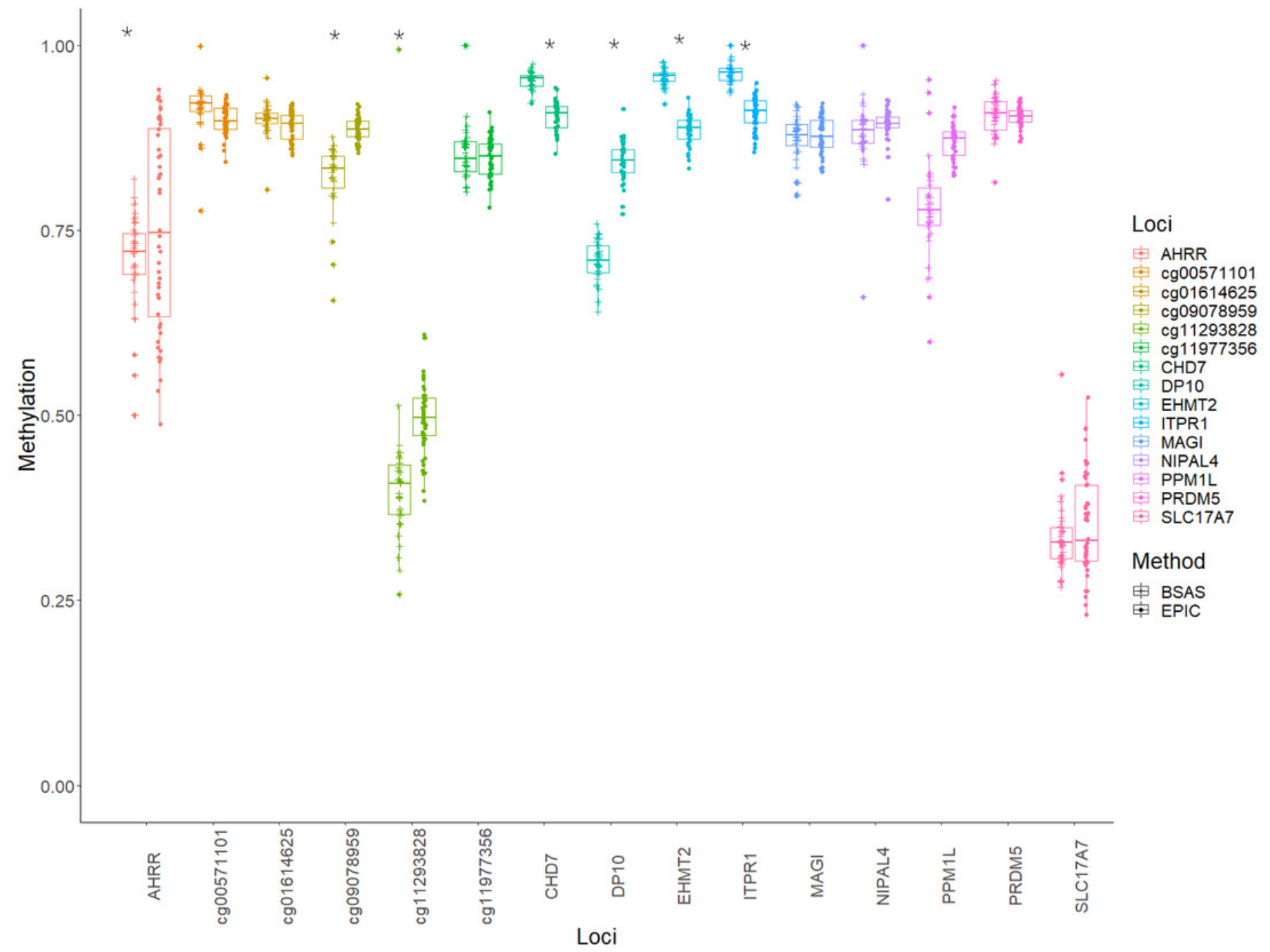


Figure 3

Figure 3 Average methylation at the 15 loci for controls

Figure 3- Average methylation for control individuals across the 15 loci assessed using EPIC and BSAS . * represent those loci with significant differences in average methylation between EPIC and BSAS.

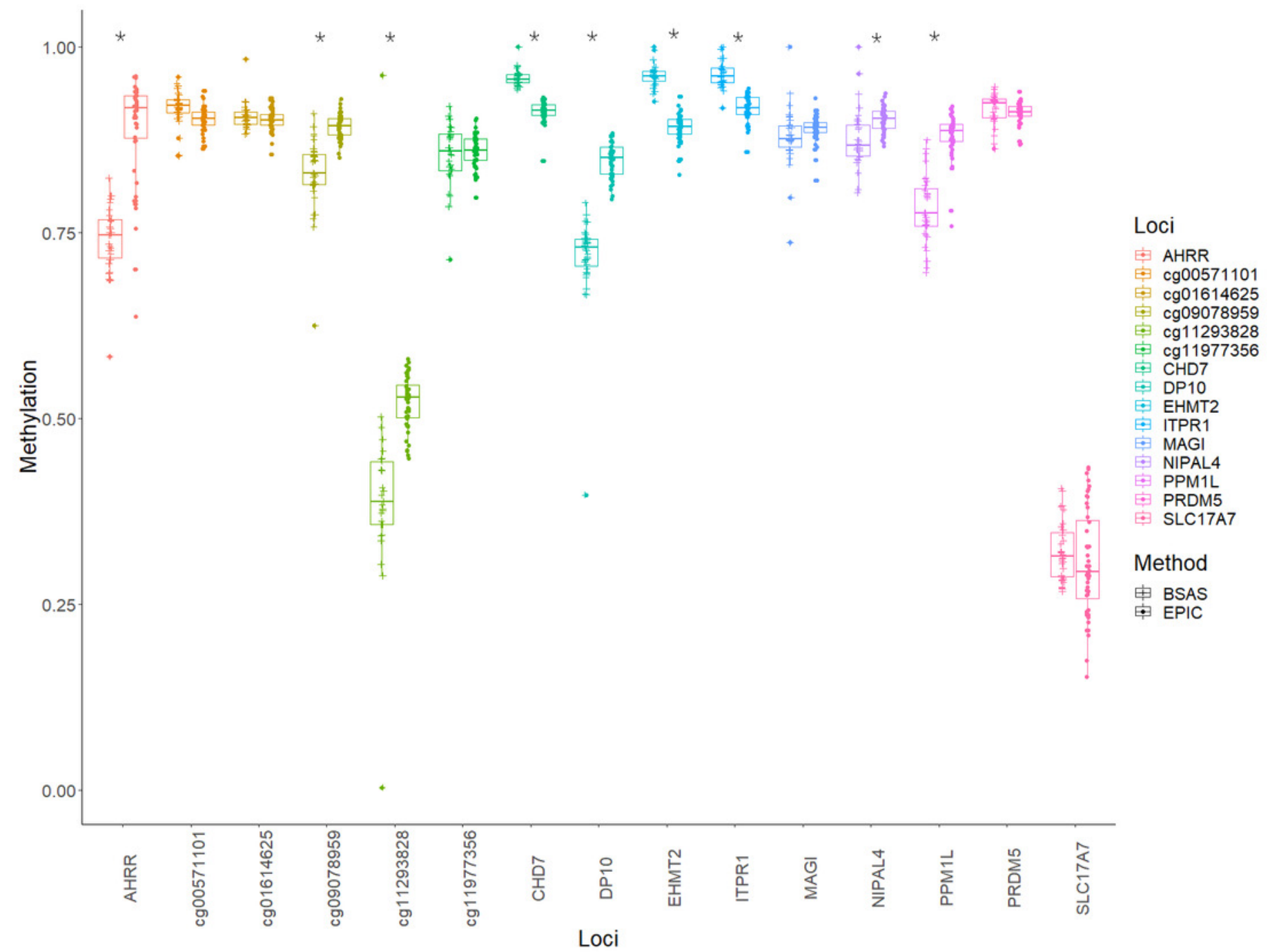

